\title{
Historia natural del carcinoma hepatocelular en una cohorte de pacientes de un hospital comarcal
}

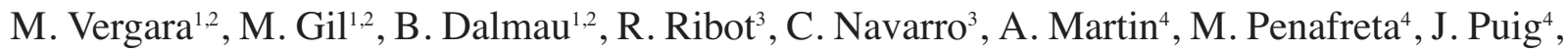 \\ J. Martin ${ }^{4}$, J. Perendreu ${ }^{4}$, J. Falcó ${ }^{4}$ y R. Bella ${ }^{5}$ \\ ${ }^{1}$ Unidad de Enfermedades Digestivas. ${ }^{2}$ CIBER HEPAD. Instituto de Salud Carlos III. ${ }^{3}$ Servicio de Medicina Interna. \\ ${ }^{4}$ Unidad de Diagnóstico de Alta Tecnología. ${ }^{5}$ Servicio de Anatomía Patológica. Corporació Sanitària Parc Tauli. \\ Sabadell, Barcelona
}

\section{RESUMEN}

Introducción: el carcinoma hepatocelular $(\mathrm{CHC})$ es una neoplasia con una elevada incidencia y una alta mortalidad.

Objetivo: nuestro objetivo es la descripción de la historia natural de una cohorte de pacientes con $\mathrm{CHC}$ e identificar variables asociadas a supervivencia.

Material y métodos: estudio retrospectivo y descriptivo de pacientes diagnosticados de CHC entre 1995-2002. Las variables cualitativas se expresaron mediante frecuencias y porcentajes y las cuantitativas con mediana y desviación típica. La supervivencia se estimó mediante el método de Kaplan Meyer y la prueba de Log Rank.

Resultados: se analizaron un total de 154 pacientes con $\mathrm{CHC}$ La ratio hombre-mujer fue de 2,9/1. La edad media de $68 \pm 9$ años. El 82\% de los pacientes fueron exitus en un tiempo medio de seguimiento de 28 meses. La mediana de supervivencia fue de 21,5 meses (IC 95\%: 16,98-26,04). Se realizó tratamiento con intención curativa en un $40,3 \%$ y el resto tratamiento paliativo. Las variables asociadas a la supervivencia fueron: presencia o no de ascitis, el número de lesiones en el momento del diagnóstico y si el tratamiento realizado fue con intención curativa o no. No hubieron diferencias estadísticamente significativas respecto a: sexo, edad, etiología de la cirrosis y estadio de Child en el momento del diagnóstico.

Conclusiones: los factores asociados a una menor supervivencia en los pacientes con carcinoma hepatocelular fueron la presencia de ascitis y el número de lesiones en el momento del diagnóstico. La realización de tratamiento con intención curativa se asocia a una mayor supervivencia.

Palabras clave: Carcinoma hepatocelular. Cirrosis hepática. Screening. Supervivencia. Ascitis.

\section{ABSTRACT}

Background: hepatocellular carcinoma (HCC) is a cancer with high incidence and mortality.

Objective: our aim was to describe the natural history of a patient cohort with HCC, and to identify the factors associated with survival.

Patients and methods: a retrospective and descriptive study of patients diagnosed with HCC between 1995 and 2002. Qualitative variables were expressed as frequencies and percentages. Quantitative variables were expressed as medians and standard deviations. Survival was calculated using the Kaplan-Meier method and log rank.

Results: a total of 154 patients were analyzed. The men-towomen ratio was $2.9 / 1$. Mean age was $68 \pm 9$ years. $82 \%$ of patients died during a median follow-up of 28 months. Median survival was 21.5 months (95\% CI: 16.98-26.04). Curative treatment was done in $40.3 \%$ of diagnosed patients, and $59.7 \%$ of patients received palliative treatment. Factors associated with survival were: ascites, number of lesions at diagnosis, and curative treatment. No statistical differences were found for the next factors: age, sex, etiology of cirrhosis, and Child-Pugh stage at diagnosis.

Conclusions: factors associated with low survival in patients with HCC were ascites and number of lesions. Curative treatment is associated with a higher survival when compared to palliative treatment.

Key words: Hepatocellular carcinoma. Liver cirrhosis. Screening. Survival. Ascites.

Vergara M, Gil M, Dalmau B, Robot R, Navarro C, Martin A, Penafreta M, Puig J, Martin J, Perendreu J, Falcó J, Bella R. Historia natural del carcinoma hepatocelular en una cohorte de pacientes de un hospital comarcal. Rev Esp Enferm Dig 2008; 100: 682-687.

Soporte financiero: Este estudio ha sido financiado en parte por el Instituto de Salud Carlos III (C03/02 y PI 05/1157 y CIBEREHD).

Recibido: $12-06-08$

Aceptado: 15-07-08.

Correspondencia: Mercedes Vergara. CIBEREHD - Unitat de Malalties Digestives. Hospital de Sabadell. Institut Universitari Parc Taulí. Universitat Autònoma de Barcelona. Parc Taulí, s/n. 08208 Sabadell, Barcelona. e-mail: mvergara@tauli.cat 


\section{INTRODUCCIÓN}

El carcinoma hepatocelular (CHC) es un tumor muy prevalente. Su incidencia anual es de un millón de casos en el mundo (1), siendo la quinta causa de cáncer más frecuente en el mundo y la tercera causa con mayor mortalidad $(1,2)$. Afecta de forma casi exclusiva a los pacientes con cirrosis hepática, con una incidencia que varía entre el 3-6,5\% anual por año de seguimiento (2), siendo la causa más frecuente de mortalidad en estos pacientes. Por ello, la cirrosis hepática es considerada una enfermedad premaligna.

La patogenia del CHC es multifactorial, con una elevada asociación con la presencia de hepatitis viral crónica, consumo de alcohol, exposición a toxinas hepáticas, así como alteraciones genéticas como la hemocromatosis o la deficiencia de alfa1-antitripsina (3). Como principales factores de riesgo para el desarrollo de CHC se han identificado niveles altos de alfa-fetoproteína $(2,4,5)$, sexo masculino, hepatitis $\mathrm{B}$, hepatitis $\mathrm{C}$, abuso de alcohol y trasfusiones previas $(2,5)$.

En España, García Torres y cols., en un estudio epidemiológico prospectivo realizado a lo largo del año 2000, hallaron que la incidencia de CHC era de 8,2/100.000 habitantes siendo la etiología predominante el virus de la hepatitis $\mathrm{C}$ (6). Otro estudio prospectivo realizado en nuestro país, mostró que las cuatro variables independientes para el desarrollo de $\mathrm{CHC}$ eran una edad superior a 55 años, la positividad para la hepatitis $\mathrm{C}$, plaquetas por debajo de $75 \times 10^{3} / \mathrm{mm}^{3}$ y un tiempo de protombina $<75 \%$ (7). Este estudio incluyó únicamente pacientes entre 40 y 65 años que son los que se benefician más del tratamiento. No obstante, en la práctica clínica diaria la población con CHC supera en muchos casos la edad de 65 años atendiendo a la larga historia natural de la enfermedad.

El screening de CHC se realiza en los pacientes diagnosticados previamente de cirrosis hepática, ya que representan un grupo de riesgo para desarrollar lesiones malignas hepáticas (8). Las características de estos pacientes son ideales para la realización del screening dado que se trata de una población diana bien establecida, con herramientas no invasivas y de bajo coste y con tratamientos curativos disponibles $(8,9)$.

El objetivo de nuestro estudio fue analizar la historia natural del CHC en nuestra área y la influencia de los diferentes factores implicados en el diagnóstico y tratamiento.

\section{MATERIAL Y MÉTODOS}

\section{Pacientes del estudio}

Se introdujeron de forma retrospectiva todos los pacientes diagnosticados de CHC entre el periodo de 19952002 en nuestra Unidad de Hepatología de la Corporació Sanitària Parc Taulí. Las historias clínicas de los pacien- tes fueron exhaustivamente revisadas, siendo incluidos un total de 154 pacientes. A todos los pacientes se les determinaron serologías de hepatitis crónica B y C, así como los anticuerpos del VIH. El hospital es centro de referencia en un área que comprende un total de 400.000 habitantes. Todos los pacientes diagnosticados de cirrosis hepática y que siguen controles en nuestra Unidad se les realiza screening de carcinoma hepatocelular de forma semestral con una determinación analítica de alfa-fetoproteína y una ecografía abdominal. Asimismo, todos los pacientes que son diagnosticados ambulatoriamente de $\mathrm{CHC}$ son remitidos posteriormente a nuestra Unidad.

\section{Variables del estudio}

Estudio descriptivo y retrospectivo en el que se recogieron variables demográficas, clínicas, bioquímicas, radiológicas y de anatomía patológica de todos los pacientes diagnosticados.

El diagnóstico de cirrosis hepática se realizó por la presencia de una primera descompensación de la hepatopatía conocida (ascitis, encefalopatía hepática o hemorragia digestiva alta por hipertensión portal), por criterios analíticos y ecográficos según el índice pronóstico validado en nuestra Unidad (10), por biopsia hepática o cuando el hepatocarcinoma había sido la causa de debut de la cirrosis hepática. Además, a todos los pacientes se les realizó serología de virus $\mathrm{C}$ y virus $\mathrm{B}$, metabolismo del hierro, alfa1 antitripsina, autoanticuerpos ANA y AMA para determinar la etiología de la hepatopatía de base.

Una vez diagnosticado el CHC se determinó si el paciente estaba en programa de screening, y si ello comporto un pronóstico y evolución diferente.

Los criterios diagnósticos del CHC fueron: una biopsia hepática compatible, dos métodos de imagen compatibles en lesiones $>2 \mathrm{~cm}$ o una prueba de imagen junto a una alfa-fetoproteína $>100 \mathrm{Ui} / \mathrm{l}$ en pacientes cirróticos, según los criterios previamente establecidos por la EASL (11). Se determinaron los datos bioquímicos de hemograma y biología hepática en el momento de diagnóstico del CHC y la función hepática de base según la clasificación de Child-Pugh (12).

El tratamiento del CHC realizado se dividió según el paciente presentase un $\mathrm{CHC}$ potencialmente curativo (cirugía resectiva, trasplante hepático, tratamiento percutáneo o cirugía resectiva) o paliativo (embolización o sintomático). El tratamiento a aplicar en cada paciente se acordó en comité de tumores del hospital.

Se identificó la fecha de exitus y la causa del mismo. Esta última variable se obtuvo de las historias clínicas.

\section{Análisis estadístico}

El análisis estadístico fue realizado usando Chi-cuadrado, test de Fisher, t de Student y test de ANOVA cuan- 
do estuvo indicado. El método de Kaplan Meier se usó para determinar la probabilidad de supervivencia acumulada después del diagnóstico de CHC. La comparación de las curvas de supervivencia obtenidas fue realizada mediante long rank test. Para el análisis de los datos se utilizó el paquete estadístico SPSS 15.0 para Windows (SPSS Inc., Chicago, EE. UU.).

\section{RESULTADOS}

\section{Características de los pacientes}

\section{Datos demográficos}

Se diagnosticaron un total de 154 pacientes entre enero de 1992 y diciembre de 2002, $115(74,7 \%)$ hombres y 39 $(25,3 \%)$ mujeres. Todos los pacientes, excepto uno, tenían cirrosis hepática. La edad media en el momento de diagnóstico de la cirrosis hepática fue de $64 \pm 12$ años. El número de casos por año fue inicialmente bajo, pero aumentando progresivamente. Así el número de casos fue 9 en el año 1995, 9 en el año 1996, 20 en el año 1997, 22 en 1998, 22 en 1999, 20 en 2000, 24 en 2001 y 28 en 2002.

\section{Características de la cirrosis hepática de base}

Las características de la cirrosis hepática de base se especifican en la tabla I. La mayoría de pacientes fue diagnosticado de cirrosis hepática por biopsia hepática o bien por el índice pronóstico. La etiología más frecuente fue la hepatitis por virus $\mathrm{C}$ o la combinación de hepatitis $\mathrm{C} \mathrm{y}$ alcohol en un $69,5 \%$ de los pacientes. Veintitrés $(17,3 \%)$ pacientes continuaban con un consumo excesivo de alcohol (> $60 \mathrm{~g}$ al día) en el momento del diagnóstico del

\begin{tabular}{|c|c|}
\hline Método diagnóstico & $\begin{array}{l}\text {-Descompensación de su hepatopatía: } 15 \text { (9,7\%) } \\
\text {-Por índice pronóstico: } 53(34,4 \%) \\
\text {-Biopsia hepática: } 58 \text { (37,7\%) } \\
\text {-A raíz del diagnóstico del CHC: } 28(18,1 \%)\end{array}$ \\
\hline Etiología de la cirrosis & $\begin{array}{l}\text {-Hepatitis crónica por virus C: } 79(51,3 \%) \\
\text {-Hepatitis crónica por virus B: } 11(7,1 \%) \\
\text {-Hepatitis crónica por VHC y alcohol: } 28 \text { (18,2\%) } \\
\text {-Alcohol } 28 \text { (18,2\%) } \\
\text {-Criptogenética: } 10(6,4 \%) \\
\text {-Hemocromatosis: } 2(1,3 \%)\end{array}$ \\
\hline Descompensaciones previas & $\begin{array}{l}\text {-Ascitis: } 56(36,6 \%) \\
\text {-Hemorragia digestiva alta por varices: } 27(17,6 \%) \\
\text {-Encefalopatía hepática precipitante: } 31(20,2 \%) \\
\text {-Peritonitis bacteriana espontánea: } 11(7,1 \%)\end{array}$ \\
\hline Tratamiento de base & $\begin{array}{l}\text {-Diuréticos: } 68(43,9 \%) \\
\text {-Lactulosa } 22(14,3 \%) \\
\text {-Beta-bloqueantes: } 40 \text { (26\%) }\end{array}$ \\
\hline
\end{tabular}

CHC, mientras que el resto se mantenía abstinente o con consumos inferiores a $20 \mathrm{~g}$ al día. Ningún paciente daba positivo en VIH. Más de la mitad de los pacientes no había presentado nunca una descompensación previa de su hepatopatía de base, pero cuando la habían presentado lo más frecuente fue una descompensación ascítica $(36,6 \%)$, por lo que casi la mitad de los pacientes de la serie $(43,9 \%)$ estaban en tratamiento con diuréticos previamente al diagnóstico de $\mathrm{CHC}$.

\section{Diagnóstico del CHC}

La edad de los pacientes en el momento del diagnóstico del CHC fue de 68,3 \pm 9,3 años. Sesenta y ocho de ellos (44\%) seguían cribaje periódico cada seis meses con determinación de alfa-fetoproteína y ecografía bianual. El resto de pacientes no lo seguían debido a no estar previamente diagnosticados de cirrosis -42 pacientes $(27 \%)$-, no tener criterios para el screening (Child C -9 pacientes- o enfermedad concomitante grave -2 pacientes-) o no seguir controles en una unidad especializada $(21 \%)$.

Ochenta y seis pacientes $(65,6 \%)$ presentaban un estadio Child A en el momento del diagnóstico. Treinta y seis $(27,5 \%)$ de ellos eran Child B y $9(6,9 \%)$ eran Child C.

En el momento del diagnóstico, 41 pacientes presentaban valores de alfa-fetoproteína superiores a $100 \mathrm{UI} / \mathrm{l}$, siendo la media de $3090 \mathrm{UI} / 1 \pm 5555$ (101-27656). El $72 \%$ de los pacientes tenían valores de su alfa-fetoproteína normal en el momento del diagnóstico.

Noventa y un pacientes (58\%) fueron diagnosticados del CHC por biopsia hepática, 39 (25,3\%) por dos métodos de imagen compatibles y $24(15 \%)$ por un método de imagen y una alfa-fetoproteína $>100$ UI/l. No se encontraron diferencias significativamente estadísticas en cuanto a la edad de diagnóstico, número de pacientes en screening o estadio de Child dependiendo de la etiología de la cirrosis hepática de base o dependiendo del consumo de alcohol activo o no en el momento del diagnóstico del CHC.

\section{Características del CHC en el momento del diagnóstico}

En el momento del diagnóstico, $86(55,8 \%)$ presentaban una única LOEH en las pruebas de imagen, $19(12 \%)$ dos LOEH y el resto de pacientes $-49(31,8 \%)$ - presentaban mas de dos LOEH, siendo considerado un CHC multifocal. El tamaño del CHC según la función hepática se visualiza en la tabla II.

Tabla II. Tamaño del CHC en el momento del diagnóstico en relación con la función hepática

\begin{tabular}{lccc}
\hline & 1 LOEH $\leq 30 \mathrm{mmHg}$ & 2 LOEH $>30 \mathrm{mmHg}$ & Multifocal \\
\hline Child A & 43 & 11 & 31 \\
Child B & 12 & 3 & 20 \\
Child C & 2 & 2 & 5 \\
\hline
\end{tabular}


De los pacientes con una única LOEH, 57 mostraban una $\mathrm{LOEH} \leq 30 \mathrm{~mm}$ de diámetro, el resto de pacientes presentaban lesiones más grandes o multifocales. El tamaño de las LOEH en referencia a la función hepática en el momento del diagnóstico se refleja en la tabla II.

No hubo diferencias en el tamaño o en el número de lesiones en el momento del diagnóstico entre los pacientes dependiendo de la etiología de la cirrosis hepática o en función del consumo de alcohol activo.

\section{Tratamiento realizado en el carcinoma hepatocelular}

El tratamiento aplicado se subdividió en dos grupos: tratamientos con intención curativa (resección quirúrgica, trasplante hepático y tratamiento percutáneo) y tratamientos paliativos (embolización hepática, tratamiento sintomático y nuevos tratamientos) (13) (Tabla III). Del total de la muestra incluida 111 eran exitus en el momento de la revisión.

A un $41,6 \%$ de los pacientes se les realizó un tratamiento curativo de primera intención, el resto de los pacientes recibió tratamiento paliativo.

Tabla III. Tratamiento realizado a los pacientes diagnosticados de carcinoma hepatocelular

\begin{tabular}{lcc}
\hline & $n$ & $\%$ \\
\hline Trasplante hepático & 11 & 7,1 \\
Tratamiento percutáneo & 41 & 26,6 \\
Resección quirúrgica & 10 & 6,5 \\
Embolización hepática & 11 & 7,1 \\
Tratamiento sintomático & 73 & 46,7 \\
Ensayos clínicos & 9 & 5,8 \\
\hline
\end{tabular}

\section{Evolución de los pacientes}

En el momento de analizar los datos $111(71,4 \%)$ pacientes habían fallecido, 23 (14,9\%) de ellos continuaban vivos y $20(13,6 \%)$ habían sido perdidos de seguimiento. La principal causa de exitus fue el propio $\mathrm{CHC}$ en 47 pacientes $(30,5 \%), 14(9,1 \%)$ de ellos fallecieron a consecuencia de insuficiencia hepatocelular como resultado de una cirrosis hepática avanzada. El resto fallecieron de otras causas no hepáticas $16(10,4 \%)$ o de complicaciones de la propia cirrosis hepática $9(5,8 \%)$ (PBE o HDA). En $25(16,2 \%)$ pacientes la causa del exitus no pudo determinarse con exactitud.

La mediana de supervivencia del total de los pacientes fue de 21,51 meses con IC 95\% (16,98-26,04).

Los pacientes que realizaron un tratamiento con intención curativa presentaron una supervivencia de 37,7 meses IC $(21,8-53,6)$, mientras que en los pacientes con tratamiento paliativo la supervivencia fue de 12,01 meses con IC 95\% $(8,2-15,7)(\mathrm{p}<0,0001)$.
La mediana de supervivencia en los pacientes en programa de screening fue de 25,39 IC $(13,09-37,70)$, mientras que en los que no lo seguían presentaron una mediana de supervivencia de 19,34 IC $(9,63-29,05)(\mathrm{p}=0,21)$.

$\mathrm{Si}$ analizamos la supervivencia según el tamaño tumoral en el momento del diagnóstico, los pacientes con tumor $<30 \mathrm{~mm}$ presentan una supervivencia de 65,43 meses IC 95\% (28-102) comparado con aquellos que presentan inicialmente un tumor $>30 \mathrm{~mm}$ o multifocal con una supervivencia de 18,7 meses IC 95\% (12-24) ( $p=0,001)$. El número de lesiones ocupantes de espacio en el momento del diagnóstico también determinó la supervivencia. Una única LOEH tuvo una supervivencia de 26,91 IC 95\% (21,36$32,46)$. Dos LOEH tuvieron una supervivencia de 30,30 IC 95\% (14,28-46,31). La presencia CHC multifocal tuvo una supervivencia de 10,20 IC 95\% (3,89-16,51) ( $p<0,05)$.

La función hepática en el momento del diagnóstico también determinó la supervivencia, así los pacientes con estadio Child A en el momento de ser diagnosticados del CHC presentaron una supervivencia de 27,14 meses IC $95 \%(20,32-33,96)$. La supervivencia en los pacientes con estadiaje Child B disminuyó hasta los 17,53 meses IC 95\% (10,57-24,50). Si los pacientes eran Child C la supervivencia fue únicamente de 8,93 IC 95\% (7,52$12,35)$ sin que alcance diferencia significativa $(p=0,8)$.

No se hallaron diferencias estadísticas en la supervivencia cuando se analizaron otros factores como el sexo; sexo femenino 20,95 IC $95 \%(12,26-29,65)$ o masculino 21,51 $95 \%$ (16,24-26,79), la etiología de la cirrosis hepática o la edad en el momento del diagnóstico siendo en los pacientes menores de 65 años de 22,66 CI 95\% $(16,50-28,83)$ o de 18,78 CI 95\% (10,10-27,46) en los mayores de 65 años.

La presencia de ascitis en el momento del diagnóstico fue un factor relevante en la supervivencia. Así, los pacientes con ascitis presentaron una supervivencia de 8,45 IC $95 \%(5,55-11,35)$ mientras que los pacientes sin ascitis fue de 26,91 IC 95\% (21,41-32,41) ( p < 0,005). Ni la presencia de encefalopatía hepática en el momento del diagnóstico del $\mathrm{CHC}$ ni la HDA por varices esofágicas modificó la supervivencia en los pacientes (Tabla IV).

Tabla IV. Supervivencia de los pacientes en función de diferentes factores asociados

\begin{tabular}{lcc}
\hline Factores asociados & Mediana de supervivencia $(95 \%$ IC) & $p$ \\
\hline $\begin{array}{l}\text { Screening/no screening } \\
\text { Tratamiento intención }\end{array}$ & $25-4(21,8-53,6) / 12,0(8,2-15,7)$ & $p=n s$ \\
$\quad$ curativa/paliativo & $37,7(21,8-53,6<9 / 12,0(8,2-15,7)$ & $p<0,05$ \\
LOEH $\leq 30$ mm / LOEH & & \\
$>30$ mm & $65,4(28-102) / 18,7(12-24)$ & $p<0,05$ \\
1 LOEH/2 LOEH / multifocal & $26,9(21,3-32,4) / 30,3(14,2-46,3) /$ & \\
& $10,1(3,8-16,5)$ & $p<0,05$ \\
Child A/Child B / Child C & $27,1(20,3-34,0) / 17,5(10,6-24,5) / 7,9$ & \\
& $(7,5-12,3)$ & $p=n s$ \\
Hombre/mujer & $21,5(16,2-26,8) / 20,9(12,2-29,6)$ & $p=n s$ \\
$\geq 65$ años/<65 años & $18,7(10,1-27,5) / 22,6(16,5-28,8)$ & $p=n s$ \\
Ascitis/no ascitis & $7,4(5,5-11,3) / 26,9(21,4-32,4)$ & $p<0,05$ \\
\hline
\end{tabular}


Al realizar un análisis multivariado de todos los factores se halló como significativos la presencia de ascitis y el número de lesiones en el momento del diagnóstico como factor predictivo de supervivencia.

\section{DISCUSIÓN}

Numerosos estudios han analizado la incidencia y los factores pronósticos asociados al hepatocarcinoma El CHC es la quinta causa de neoplasia más frecuente (1), siendo la etiología más prevalente en Europa la infección crónica por el virus C (14). Más de la mitad de los pacientes de nuestra cohorte presentaban la infección por la hepatitis $\mathrm{C}$ sola o asociada con el alcohol. Es importante hacer notar la baja incidencia de la infección por hepatitis $\mathrm{B}$ en nuestro medio, al igual que en otro estudio previo realizado en un área cercana (15). Todos los casos, excepto uno, de $\mathrm{CHC}$ diagnosticados se han desarrollado sobre un hígado cirrótico mostrando la teoría de que es la cirrosis, el principal factor oncogénico y no la etiología como se había postulado previamente. A pesar de ello en la literatura se siguen reportando casos de $\mathrm{CHC}$ en pacientes con hepatopatia crónica sin signos de cirrosis (16).

Nuestro estudio tiene como limitaciones la recogida de datos retrospectiva y un periodo de inclusión prolongado. No obstante, las variables del estudio son objetivas y recogidas de forma sistemática en la historia clínica para que la calidad de los datos analizados sea alta. Asimismo, todos los pacientes en el momento de ser diagnosticados eran introducidos en una base de datos por lo que se obvia el sesgo de no haber sido adecuadamente contabilizados. Dado que los pacientes incluidos están diagnosticados en un periodo largo de tiempo no se ha recogido el estadiaje del CHC dado que a lo largo de los años hubo un cambio en la clasificación y esta no fue uniforme a lo largo de los años.

Tradicionalmente se ha discutido en la literatura médica la utilidad del screening del CHC para mejorar la supervivencia. Actualmente la alfa-fetoproteína elevada se considera un factor de riesgo de desarrollo de $\mathrm{CHC}$, por tanto puede ser utilizada para determinar los pacientes en riesgo de adquirir $\mathrm{CHC}$, sin embargo no parece adecuada como screening (11). Estudios previos han hallado pacientes diagnosticados de $\mathrm{CHC}$ con niveles altos de alfafetoproteína en más de la mitad de los pacientes $(17,18)$, si bien en nuestro estudio este porcentaje era únicamente de un $27 \%$. Se ha demostrado que estos niveles estan en relación con el tamaño tumoral y por lo que son de escasa utilidad en el diagnóstico precoz de la enfermedad (19).

Nuestro estudio muestra que los pacientes en programa de screening se diagnostican antes, pero no que la supervivencia cambie con la realización de un screening. Xhang y cols. reclutaron a 18.816 pacientes y concluyeron que el screening reducía la mortalidad un $37 \%$ (20). Las guías clínicas recomiendan la realización de screening en pacientes en estadio Child-Pugh A, desaconsejan si se encuentra en Child C y queda en duda si es Child B (21). Recientemente un estudio demuestra que la supervivencia es mayor en los pacientes en programa de screening con una función hepática intermedia (Child Pugh B), sin embargo no hay ninguna utilidad cuando los pacientes presentan una cirrosis evolucionada (22). Nuestro estudio indirectamente también determina que la función hepática es importante a la hora de realizar screening y que en los pacientes con enfermedad hepática evolucionada no es útil, dado que el estadio evolutivo de la cirrosis disminuye la supervivencia. Es importante señalar que en nuestra población de pacientes más de la mitad presentaban una función hepática conservada en el momento del diagnóstico.

Hasta hace poco, el diagnóstico de estos tumores se realizaba casi universalmente o bien en fases avanzadas, cuando no era posible aplicar tratamientos con capacidad curativa, o bien en fase precoces cuando la enfermedad hepática se descompensaba por otro motivo. En cualquier caso, el pronóstico era muy malo, con una supervivencia a los cinco años del 0-10\% (23). El CHC es un tumor de mal pronóstico, que en el momento del diagnóstico sólo un $30-40 \%$ de los pacientes pueden optar a tratamiento potencialmente curativo (24). Este porcentaje es similar al hallado en nuestro estudio en donde un $41 \%$ de nuestros pacientes optaron a tratamiento con intención curativa. Estos datos son indicativos de que las herramientas de las que disponíamos para el diagnóstico precoz del CHC eran eficaces. Nuestro estudio no ha recogido la presencia de síntomas específicos asociados a la enfermedad neoplásica como la astenia, anorexia o pérdida de peso, dado que al ser una recogida retrospectiva estos datos eran subjetivos y difíciles de cuantificar. Su presencia, suele asociarse a enfermedad avanzada, fuera de opciones terapéuticas (25). Estudios recientes realizados en nuestra área que han analizado también datos pronósticos de mala evolución no han hallado diferencias en la supervivencia en aquellos pacientes con o sin ascitis (26). Nuestros resultados muestran que la presencia de ascitis en el momento del diagnóstico determina una menor supervivencia, ello probablemente es debido por un lado a que nuestra cohorte de pacientes es más amplia (con más potencia estadística) y, por otro, a que en este estudio previamente mencionado únicamente se han analizado pacientes en tratamiento paliativo y por tanto, de entrada con mal pronóstico. La ascitis es un parámetro de mala función hepática que forma parte de la clasificación de Child-Pugh y consecuentemente es razonable que se halle relacionado con una peor supervivencia en el momento del diagnóstico.

La supervivencia obtenida en los pacientes con tratamiento curativo es similar a la de otros estudios publicados recientemente (27), lo que avala la calidad de los datos recogidos.

En conclusión, en el hepatocarcinoma, los factores asociados a una menor supervivencia en los pacientes con carcinoma hepatocelular fueron la presencia de asci- 
tis y un mayor número de lesiones en el momento del diagnóstico. La realización de tratamiento con intención curativa se asocia a una mayor supervivencia.

\section{BIBLIOGRAFÍA}

1. Parkin DM, Bray F, Ferlay J, Pisani P. Estimating the world cancer burden: Globocan 2000. Int J Cancer 2001; 94: 153-6.

2. Ikeda K, Saitoh S, Koida I, Arase Y, Tsubota A, Chayama K, et al. A multivariate analysis of risk factors for hepatocellular carcinogenesis: a prospective observation of 795 patients with viral and alcoholic cirrhosis. Hepatology 1993; 18: 47-53.

3. Muntané J, González R, Ranchal I, Collado JA, López-Sánchez LM, Herencia C, et al. Mechanisms of liver cell injury. Rev Esp Enferm Dig 2007; 99: 405-10.

4. Colombo M, de Franchis R, Del Ninno E, Sangiovanni A, De Fazio $\mathrm{C}$, Tommasini M, et al. Hepatocellular carcinoma in Italian patients with cirrhosis. N Engl J Med 1991; 325: 675-80.

5. Tsukuma H, Hiyama T, Tanaka S, Nakao M, Yabuuchi T, Kitamura $\mathrm{T}$, et al. Risk factors for hepatocellular carcinoma among patients with chronic liver disease. N Engl J Med 1993; 328: 1797-801.

6. García-Torres ML, Zaragoza A, Giner R, Primo J, del Olmo JA. Incidence and epidemiological factors of hepatocellular carcinoma in Valencia during the year 2000. Rev Esp Enferm Dig 2003; 95: 385-4.

7. Velázquez RF, Rodríguez M, Navascues CA, Linares A, Pérez R, Sotorríos NG, et al. Prospective analysis of risk factors for hepatocellular carcinoma in patients with liver cirrhosis. Hepatology 2003; 37: 520-7.

8. Repiso A, Gómez RR, González de Frutos C, de AT, Sánchez Ruano JJ, Pérez Grueso MJ, et al. Angioma-like liver lesions in patients with chronic liver disease. Rev Esp Enferm Dig 2007; 99: 259-63.

9. Bruix J, Llovet JM. Prognostic prediction and treatment strategy in hepatocellular carcinoma. Hepatology 2002; 35: 519-24.

10. Obrador BD, Prades MG, Gomez MV, Domingo JP, Cueto RB, Rue $\mathrm{M}$, et al. A predictive index for the diagnosis of cirrhosis in hepatitis $\mathrm{C}$ based on clinical, laboratory, and ultrasound findings. Eur J Gastroenterol Hepatol 2006; 18: 57-62.

11. Bruix J, Sherman M, Llovet JM, Beaugrand M, Lencioni R, Burroughs AK, et al. Clinical management of hepatocellular carcinoma. Conclusions of the Barcelona-2000 EASL conference. European Association for the Study of the Liver. J Hepatol 2001; 35: 421-30.

12. Pugh RN, Murray-Lyon IM, Dawson JL, Pietroni MC, Williams R. Transection of the oesophagus for bleeding oesophageal varices. Br J Surg 1973; 60: 646-9.
13. Dalhoff K, Dancey J, Astrup L, Skovsgaard T, Hamberg KJ, Lofts FJ, et al. A phase II study of the vitamin D analogue Seocalcitol in patients with inoperable hepatocellular carcinoma. Br J Cancer 2003; 89: 252-7.

14. Trinchet JC, Ganne-Carrie N, Nahon P, N'kontchou G, Beaugrand M. Hepatocellular carcinoma in patients with hepatitis $\mathrm{C}$ virus-related chronic liver disease. World J Gastroenterol 2007; 13: 2455-60.

15. Calvet X, Bruix J, Bru C, Gines P, Vilana R, Sole M, et al. Natural history of hepatocellular carcinoma in Spain. Five year's experience in 249 cases. J Hepatol 1990; 10: 311-7.

16. Lucero Pizones JA, Salman MZ, Jou MC, Novella DM, Garcia Gutierrez MJ. Hepatocellular carcinoma associated with non-cirrhotic chronic hepatitis C. Rev Esp Enferm Dig 2007; 99: 364-5.

17. Herold C, Reck T, Fischler P, Ott R, Radespiel-Troeger M, Ganslmayer M, et al. Prognosis of a large cohort of patients with hepatocellular carcinoma in a single European centre. Liver 2002; 22: 23-8.

18. Nzeako UC. Etiologic factors and clinical presentation of hepatocellular carcinoma: differences between cirrhotic and noncirrhotic Italian patients. Cancer 1995; 76: 915.

19. Bruix J, Calvet X, Vilana R. Increased alphafetoprotein levels in hepatocellular carcinoma: the relationship with tumor size determines its limited value in early detection. J Hepatol 2008; 9: 8-16.

20. Zhang BH, Yang BH, Tang ZY. Randomized controlled trial of screening for hepatocellular carcinoma. J Cancer Res Clin Oncol 2004; 130: 417-22.

21. Bruix J, Sherman M. Management of hepatocellular carcinoma. Hepatology 2005; 42: 1208-36.

22. Trevisani F, Santi V, Gramenzi A, Di Nolfo MA, Del PP, Benvegnu L, et al. Surveillance for early diagnosis of hepatocellular carcinoma: is it effective in intermediate/advanced cirrhosis? Am J Gastroenterol 2007; 102: 2448-57.

23. Llovet JM, Burroughs A, Bruix J. Hepatocellular carcinoma. Lancet 2003; 362: 1907-17.

24. Bruix J, Sala M, Llovet JM. Chemoembolization for hepatocellular carcinoma. Gastroenterology 2004; 127: S179-S188.

25. López PM, Villanueva A, Llovet JM. Systematic review: evidencebased management of hepatocellular carcinoma --an updated analysis of randomized controlled trials. Aliment Pharmacol Ther 2006; 23 : $1535-47$

26. Díaz SA, Nunez MO, Prieto MM, Beceiro PI, Calleja KJ, Santos $\mathrm{CL}$, et al. Prognostic factors in patients with non-active treatment of hepatocellular carcinoma. Gastroenterol Hepatol 2007; 30: 441-8.

27. Guglielmi A, Ruzzenente A, Sandri M, Pachera S, Pedrazzani C, Tasselli S, et al. Radio frequency ablation for hepatocellular carcinoma in cirrhotic patients: prognostic factors for survival. J Gastrointest Surg 2007; 11: 143-9. 